\title{
On Uncertain Granular Numbers
}

\author{
Assem A. Alsawy \\ Central Laboratory for Agricultural Expert System \\ Giza - Egypt
}

\begin{abstract}
When a quantity is measured, the outcome depends on several factors like the measuring system, the measurement procedure, the skill of the operator, the environment, and other effects. In case of inexact quantity the outcome may also depends on the uncertainty representation.

Recently inexact numbers got a lot of attention from many researchers in different fields. In this paper, we have tried to give an overview for the different representations of the inexact granular numbers. The objective of this overview is providing a certain insight into the essence of granular data representation being regarded as a framework of representing and manipulation of inexact information, and introduce a new representation of granular uncertain number to be as step in formulate a general form for all uncertain numbers.
\end{abstract}

\section{General Terms}

Granular Computing, Soft Computing, Uncertainty

\section{Keywords}

Fuzzy number, Rough number, Interval, Grey number, Vague number, Granular number

\section{INTRODUCTION}

In the real world, a lot of knowledge often comes vague, imprecise, uncertain, or probabilistic in nature. Human thinking and reasoning often contain inaccurate information originated from inherently inexact human concepts; humans can give satisfactory answers, which are probably true. Because the information people obtain is always uncertain and limited, a variety of uncertainty theories emerged. Young R.C. and Moore R E. proposed the interval number [1], [2], L.A. Zadeh proposed fuzzy mathematics [3] then he introduced a type-2 fuzzy set (and even higher types) [4] and Z. Pawlak proposed the rough sets theory [5]. In the meantime, because of limited information and knowledge, only part of system structure could be fully realized. To overcome this problem, Deng J.L. proposed the grey systems theory [6], also Atanassov suggests lntuitionistic fuzzy sets [7], and D. J. Buehrer and W.L. Gau proposed for vague set [8]. In recent years the definition of inexact number has been emerged from uncertainty set theories. Fuzzy sets, interval analysis, rough sets, grey set, lntuitionistic fuzzy sets, and vague set are all constructs falling under the same umbrella of Granular Computing which has recently emerged as a coherent conceptual and algorithmic platform aimed at the representation and processing of information granules [9].

\section{CLASSICAL SET}

Set theory was founded by a single paper in 1874 by George Cantor: "On a characteristic property of all real algebraic numbers" [10]. A set in mathematics is a collection of well defined and distinct objects, considered as an object in its own right. Sets are one of the most fundamental concepts in mathematics. Set theory is now a ubiquitous part of mathematics, and can be used as a foundation from which nearly all of mathematics can be derived. The theory of sets

\author{
Hesham A.Hefny \\ Institute of Statistical Studies and research Cairo \\ University \\ Giza - Egypt
}

based on the notion of element membership to sets has proved itself to be one of the most powerful tools of what is known as modern mathematics and it has allowed us to study modeling and development of other sciences. However, the notion of element membership to a set is a bivalent concept, governed by the values 0 (there is no membership) and 1 (there is membership) and it does not allow other set possibilities which, nevertheless, have been studied in the fields of Logic models [11]. An object in a set is called an element or member of that set. Sets are defined by a simple statement describing whether a particular element having a certain property belongs to that particular set. Classical set theory enumerates all its elements using: $A=\left\{a_{1}, a_{2}, \ldots . a_{n}\right\}$. If the elements $a_{i}(i=1,2, . . n)$ of a set $A$ are subset of universal set $X$, then the set $A$ can be represented for all elements $x \in X$ by its characteristic function

$$
\mu_{A}(x)=\left\{\begin{array}{l}
1 \text { if } x \in X \\
0 \text { otherwise }
\end{array}\right.
$$

\subsection{A number}

A number is a mathematical object used to count, label, and measure. In mathematics, the definition of number has been extended over the years to include such numbers as zero, negative numbers, rational numbers, irrational numbers, and complex numbers. A notational symbol that represents a number is called a numeral. In addition to their use in counting and measuring, numerals are often used for labels, for ordering, and for codes. In common use, the word number can mean the abstract object, the symbol, or the word for the number [12]. Now the definition of number involve the uncertainty representation and granular forms which come from uncertain sets representation such as: fuzzy set, rough set, grey set vague set ,.....etc

\section{INTERVALS (INTERVAL NUMBERS)}

One of the earliest observations of digital numerical computation was that, although most programs give highly accurate results, it can happen that rounding errors build up in such a way that none of the many decimals in the result is meaningful. A good early summary of this is Forsythe's "The Pitfalls of Numerical Computation". Instead of using a single floating-point number as approximation for the value of a real variable in the mathematical model under investigation, interval arithmetic acknowledges limited precision by associating with the variable a set of reals as possible values. For ease of storage and computation, these sets are restricted to intervals. The computation rules aim at maintaining the property of containing all possible values. In the past two decades, the interval method has been obtaining more and more attentions. Interval represents a closed bounded set of real numbers, and in interval mathematics, it is regarded as a type of number, namely interval number [13]. Real interval is a set of real numbers with the property that any number that lies between two numbers in the set is also included in the set. 
For example, the set of all numbers $x$ satisfying $3 \leq x \leq 4$ is an interval which contains 3 and 4 , as well as all numbers between them. Other examples of intervals are the set of all real numbers $\square$, the set of all negative real numbers, and the empty set. Real intervals play an important role in the theory of integration, because they are the simplest sets whose "size", "measure" or "length" is easy to define. In literature there exist two different types of notions to represent an interval. The first one assumes that an interval number is a non-fuzzy subset of the real line with a precisely defined domain on it as [14]: $A=[a, b]=\{x \in \square \mid a \leq x \leq b\} \quad$ It is an ordered pair of real numbers. This representation also assumes the following form where $m(A)$ and $w(A)$ are the center and radius of a disk mapped on the real line $A=\langle m(A), w(A)\rangle=$ $\{x \mid m(A)-w(A) \leq x \leq m(A)+w(A), m(A), w(A) \in \square\}$ Where $m(A)=\frac{1}{2}(a+b)$ and $w(A)=\frac{1}{2}(b-a)$

$\boldsymbol{A}$ can be defined again as [13]: $A=[m(A)-w(A), m(A)+w(A)]$. Interval can be represented in another way by its characteristic function as shown in figure (1), $\forall x \in A$ on $\square$

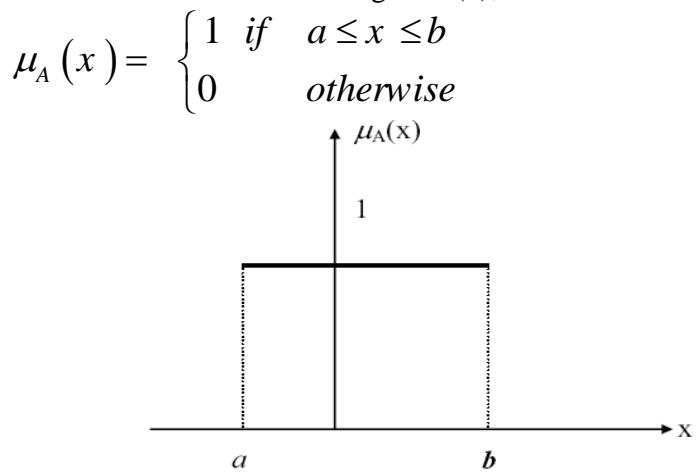

Fig 1: Interval $A=[\mathbf{a}, \mathbf{b}]$

\section{FUZZY SET}

In most of cases in our life, the data obtained for decision making are only approximately known. In 1965, Zadeh introduced the concept of fuzzy logic and fuzzy set theory to meet those problems. A logic based on the two truth values, true and false is sometimes unsuitable when describing human reasoning. Fuzzy logic provides a rich and meaningful addition to standard logic [3]. Basically, Fuzzy Logic (FL) is a multivalued logic that allows intermediate values to be defined between conventional evaluations like true/false, yes/no, high/low, etc. So we can say that Fuzzy logic is a superset of conventional (Boolean) logic that has been extended to handle the concept of partial truth values between "completely true" and "completely false". Notions like "rather tall" or "very fast" can be formulated mathematically and processed by computers, in order to apply a more human-like way of thinking in the programming of computers. Fuzzy logic is capable of handling inherently imprecise concept [16]. The very basic notion of fuzzy logic is a fuzzy set. In classical mathematics we are familiar with crisp sets. The elements which have been assigned the number 1 can be interpreted as the elements that are in the set A and the elements which have assigned the number 0 as the elements that are not in the set A. This concept is sufficient for many areas of applications, but it can easily be seen that it lacks flexibility for some applications. Different to classical sets, elements of a fuzzy set have membership degrees to that set; the degree of membership to a fuzzy set indicates the certainty (or uncertainty) that the element belongs to that set. So the fuzzy set theory is an extension of classical set theory where elements have varying degree of membership. A fuzzy set is any set that allows its members to have different degree of membership, within interval $[0,1]$. In the universal space $\mathrm{X}$ a fuzzy set $\mathrm{A}$ is written as a set of pairs $A=\left\{x, \mu_{A}(x)\right\}$ Where $\mathrm{x}$ is an element of the universal space $\mathrm{X}$, and $\mu_{A}(x)$ is the value of the membership function for element $\mathrm{x}$.

\subsection{Fuzzy number}

A fuzzy number is simply an ordinary number whose precise value is somewhat uncertain. Fuzzy numbers are the natural generalization of real, crisp numbers; they are used in statistics, computer programming, engineering, and experimental science. Dubois and Prade defined any of the fuzzy numbers as a fuzzy subset of the real line [17]. Fuzzy numbers allow us to make the mathematical model of linguistic variable on fuzzy environment. A fuzzy number is a quantity whose value is imprecise, rather than exact as is the case with "ordinary" (single-valued) numbers [18]. If a fuzzy set is convex and normalized, and its membership function is defined in $\mathrm{R}$ and piecewise continuous, it is called as fuzzy number. So, fuzzy number represents a real number interval whose boundary is fuzzy. It is expressed as a fuzzy set defining a fuzzy interval in the real number R. Since the boundary of this interval is ambiguous, the interval is also a fuzzy set. Here, we give some necessary definitions of fuzzy set theory.

Definition: A fuzzy set $A$ is called normal if there is at least one point $x \in \mathfrak{R}$ with $\mu_{A}(x)=1$.

Definition [20]: A fuzzy set $A$ on $R$ is convex if for any, $x, y$ $\in R$ and any $\lambda \in[0,1]$, we have

$$
\mu_{A}(\lambda x+(1-\lambda) y) \geq \min \left\{\mu_{A}(x), \mu_{A}(y)\right\}
$$

Definition: A fuzzy number is a fuzzy set on the real line that satisfies the Conditions of normality and convexity. A fuzzy number is a fuzzy set which has the following three properties [21]:

- A fuzzy number must be a normal fuzzy set, which means the maximum membership of any element in this number is 1.0 .

- $\quad$ All alpha levels must be closed for every $\alpha \in(0,1]$.

- The support of a fuzzy number must be bounded.

\subsection{Types of fuzzy number}

There are many forms of fuzzy number, among which the triangular fuzzy number, trapezoidal fuzzy number and Gaussian fuzzy model are the most common ones

\subsubsection{Triangular fuzzy number}

Definition: Triangular fuzzy number is a fuzzy number represented with three points as follows [19]: 
$A=\left(a_{1}, a_{2}, a_{3}\right)$ as shown in figure 2. This representation is interpreted as membership functions as follows [19]:

$$
\mu_{A}(x)=\left\{\begin{array}{lc}
0 & x<a_{1} \\
\frac{x-a_{1}}{a_{2}-a_{1}} & a_{1} \leq x<a_{2} \\
\frac{a_{3}-x}{a_{3}-a_{2}} & a_{2} \leq x<a_{3} \\
0 & x>a_{3}
\end{array}\right.
$$

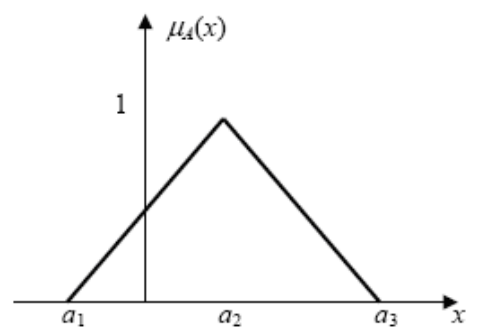

Fig 2: Triangular fuzzy number $A=\left(a_{1}, a_{2}, a_{3}\right)$

\subsubsection{Trapezoidal Fuzzy Number}

Another shape of fuzzy number is trapezoidal fuzzy number. This shape is originated from the fact that there are several points whose membership degree is maximum $(\alpha=1)$.

Definition: Trapezoidal fuzzy number is a fuzzy number represented with four points as follows: $A=\left(a_{1}, a_{2}, a_{3}, a_{4}\right)$ as shown in figure 3 . The membership function of this fuzzy number will be interpreted as follows [19]:

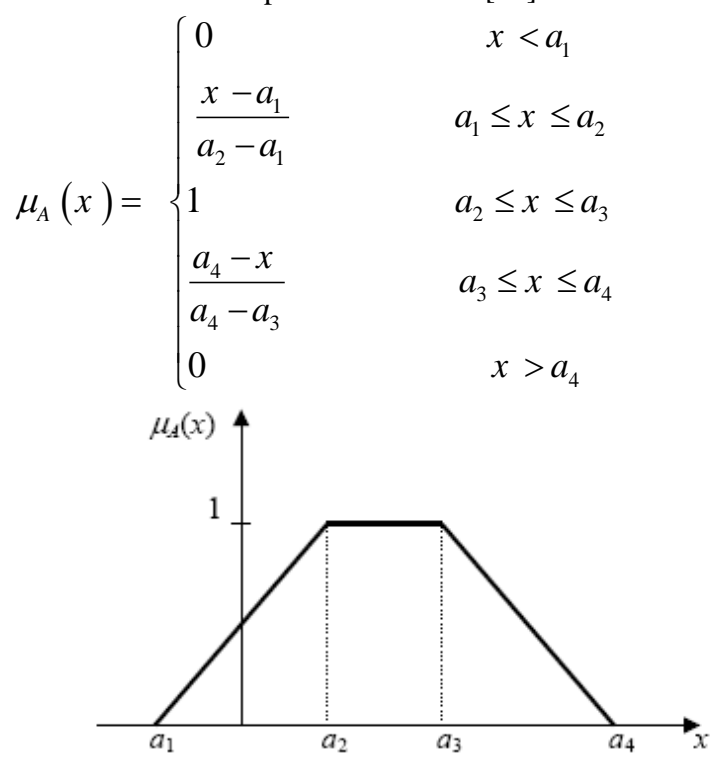

Fig 3: Trapezoidal fuzzy number $A=\left(a_{1}, a_{2}, a_{3}, a_{4}\right)$

\subsubsection{Bell Shape Fuzzy Number}

Bell shape fuzzy number is often used in practical applications and its function is defined as follows [19]:

$$
\mu(x)=\exp \left\{-1 / 2[(x-m) / \sigma]^{2}\right\} .
$$

Where $\boldsymbol{m}$ is the mean of the function, $\sigma$ is the standard deviation

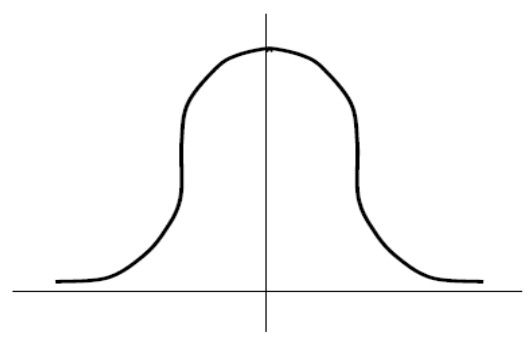

Fig 4: Bell Shape Fuzzy Number

\section{TYPE-2 FUZZY SET}

Type-2 fuzzy sets were initially defined by Zadeh (1975) [4], as an extension of the concept of an ordinary fuzzy set (henceforth called a "type-1 fuzzy set"). A type-2 fuzzy set is characterized by a fuzzy membership function, i.e. such sets can be used in situations where there is uncertainty about the membership grades themselves, e.g., an uncertainty in the shape of the membership function or in some of its parameters. Consider the transition from ordinary sets to fuzzy sets. When we can determine the membership of an element in a set as 0 or 1, we use fuzzy sets of type-1. Similarly, when the situation is so fuzzy that we have trouble determining the membership grade even as a crisp number in $[0,1]$, we use fuzzy sets of type-2 [22].

Type-2 sets can be used to translate the uncertainties in membership functions of type- 1 sets, due to the dependence of the membership functions on available linguistic and numerical information. Linguistic information such as rules from experts in general, does not give any information about the shapes of the membership functions. When membership functions are determined or tuned based on numerical data, the uncertainty in the numerical data, e.g., noise, translates into uncertainty in the membership functions. In all such cases, information about the linguistic or numerical uncertainty can be incorporated in the type- 2 framework. A Type- 2 set $\boldsymbol{A}$ is characterized by a three dimensional membership function which itself is fuzzy as follows [23]:

$A=\left\{\left((x, u), \mu_{A}(x, u)\right) \mid \forall x \in X, \forall u \in J_{x} \subseteq[0,1]\right\}$

where. $0 \leq \mu_{A}(x, u) \leq 1$

\subsection{Type-2 Fuzzy Number}

A type-1 fuzzy number (T1FN) is defined as a fuzzy set that is both normal and convex. Normality is required in order to capture the concept of a fuzzy number being a set of real numbers close to a specific crisp number, in other words when all the uncertainty about a number disappears it reduces to a crisp number. Convexity is required as it allows meaningful arithmetic operations to be performed on fuzzy sets using the well established methods from interval analysis since $\alpha$-level sets are closed intervals. Define a type-2 fuzzy number (T2FN) as a type-2 fuzzy set having a numerical domain. There is no assumption for normality has been defined for T2FN.

\subsection{Types of Type-2 fuzzy number}

There are various shapes of Type-2 fuzzy numbers; Gaussian Type-2 fuzzy number and Interval type-2 fuzzy number are illustrated in next subsections.

\subsubsection{Gaussian Type-2 fuzzy number}

A fuzzy set characterized by a Gaussian membership function with fixed mean $m$ and uncertain standard deviation that can take values in $\left[\sigma_{1}, \sigma_{2}\right]$ 


$$
\mu(x)=\exp \left\{-1 / 2[(x-m) / \sigma]^{2}\right\} ; \quad \sigma \in\left[\sigma_{1}, \sigma_{2}\right]
$$

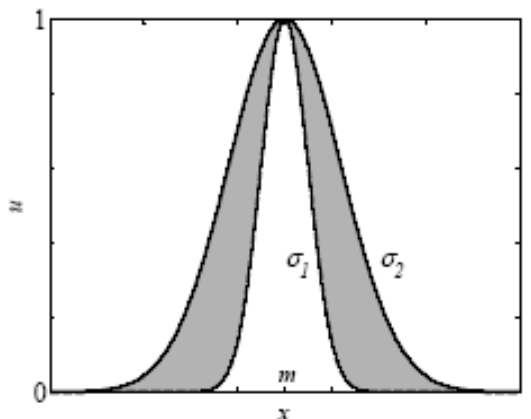

Fig 5: A Gaussian type-2 fuzzy number with uncertain standard deviation [23].

A fuzzy set characterized by a Gaussian membership function with fixed standard deviation and uncertain mean that can take values in [m1, m2].

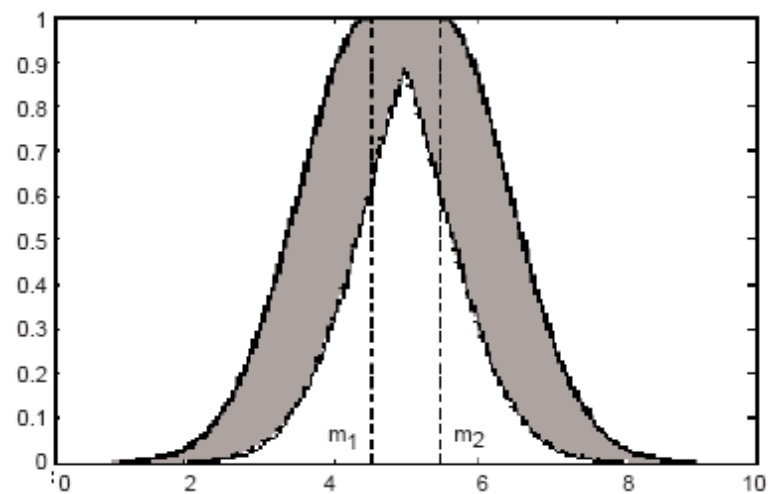

Fig 6: A Gaussian type-2 fuzzy number with uncertain mean $[23]$

\subsubsection{Interval type-2 fuzzy number}

Type-2 fuzzy sets are characterized by membership grades which are fuzzy subsets of the unit interval $[0,1]$. More precisely, the fuzzy set of type-2, $\boldsymbol{A}$, in the real line $\square$, is a vague collection of elements characterized by membership function $\mu_{A}: \square \rightarrow F([0,1])$ where $\boldsymbol{F}([0,1])$ is a set of all classical fuzzy sets in the unit interval $[0,1]$. Each $x \in \square$ is associated with a secondary membership function $f_{x} \in F([0,1])$, which is a mapping $f_{x}:[0,1] \rightarrow[0,1]$ [23].

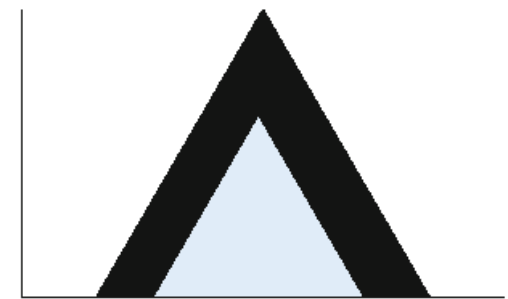

Fig 7: Interval type-2 fuzzy number

\section{ROUGH SET}

Pawlak introduced the theory of rough sets as an extension of set theory for the study of intelligent systems characterized by insufficient and incomplete information. Rough set theory can be regarded as a new mathematical tool for imperfect data analysis. That theory has found applications in many domains, such as decision support, engineering, environment, banking, medicine and others. Rough set philosophy is founded on the assumption that with every object of the universe of discourse some information (data, knowledge) is associated. Objects characterized by the same information are indiscernible (similar) in view of the available information about them. The indiscernibility relation generated in this way is the mathematical basis of rough set theory. Any set of all indiscernible (Similar) objects is called an elementary set, and forms a basic granule (atom) of knowledge about the universe. Any union of some elementary sets is referred to as a crisp (precise) set - otherwise the set is rough (imprecise, vague). Each rough set has boundary-line cases, i.e., objects which cannot be with certainty classified, by employing the available knowledge, as members of the set or its complement. Obviously rough sets, in contrast to precise sets, cannot be characterized in terms of information about their elements. With any rough set a pair of precise sets, called the lower and the upper approximation of the rough set, is associated. The lower approximation consists of all objects which surely belong to the set and the upper approximation contains all objects which possibly belong to the set. The difference between the upper and the lower approximation constitutes the boundary region of the rough set. Approximations are fundamental concepts of rough set theory [24]. In rough set theory, this indiscernability of the data points is handled using the concept of approximate sets. The upper and lower approximate sets of are denoted as $\bar{R}(X)$ and $\underline{R}(X)$, respectively, and are defined as [25]:

$$
\begin{aligned}
& \underline{R}(X)=\left\{x \in U \mid[x]_{P} \subseteq X\right\} \\
& \bar{R}(X)=\left\{x \in U \mid[x]_{P} \cap X \neq \varphi\right\}
\end{aligned}
$$

Where $[x]_{P}$ represents the equivalence class determined by $x$ with respect to $P$.

\subsection{Rough Number}

Rough numbers [26] are based on the several following notions. For the set $\square^{+}$a sequence of nonnegative real numbers $S=\left(x_{i}\right)_{i=1}^{n} \quad$ such that $x_{i}<x_{j}, i<j$ is called the categorization. The approximation space is the ordered pair $A=\left(\square^{+}, S\right)$. Every categorization $S$ of $\square^{+}$ induces partition $\pi(\mathrm{S})$ on $\square^{+}$defined as $\pi(S)=\left\{0,\left(0, x_{1}\right), x_{1}, \ldots, x_{i},\left(x_{i}, x_{i+1}\right), \ldots\right\}$ where $\left(x_{i}, x_{i+1}\right)$ denotes the open interval. $\mathrm{S}(\mathrm{x})$ denotes the block of partition $\pi(\mathrm{S}): \mathrm{x} \in \pi(\mathrm{S})$. Let $x \in\left(x_{i}, x_{i+1}\right)$, the closed interval $\bar{S}(x)=\left\langle x_{i}, x_{i+1}\right\rangle \quad$ is called the closure $\mathrm{S}(\mathrm{x}) . \mathrm{Q}(\mathrm{x})$ is the closed interval $\langle 0, x\rangle$. For a given approximation space $\mathrm{A}\left(\square^{+}, \mathrm{S}\right)$ for every $\mathrm{Q}(\mathrm{x})$ its lower and upper approximation may be defined, denoted as $S_{*}(Q(x))$ and $S^{*}(Q(x))$ respectively.

$S_{*}(Q(x))=\{y \in R+: S(y) \subseteq Q(x)\}$

$S^{*}(Q(x))=\{y \in R+: S(y) \cap Q(x) \neq \varnothing\}$

As the S-lower and S-upper approximation of the nonnegative real number $\mathrm{x}$ the following values are considered: 
$S_{*}(x)=\operatorname{Sup}\{y \in S: y \leq x\}$;

$S^{*}(x)=\operatorname{Inf}\{y \in S: y \geq x\}$.

All notions above lead to the definition of an approximation of a real number $\mathrm{x}$ on the basis of a set of real numbers

$S: S(x)=\left(S_{*}(x), S^{*}(x)\right)$. The number is exact in $A=\left(\square^{+}, S\right), A=\left(\square^{+}, S\right)$ if $S_{*}(x)=S^{*}(x)$

otherwise it is inexact (rough) in A. Every inexact number may be represented as a pair of exact numbers.

Example: let $S=\left\{x_{0}, x_{1}, \ldots, x_{n}\right\}$ be a sequence on the real line R such that $x_{0}<x_{1}<\ldots<x_{n}$

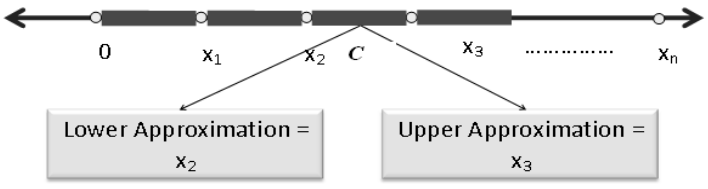

Fig 8: rough number C: $x_{2}<C<x_{3}$

\subsection{Rough real number}

Rough real number is Extended Models of Rough Numbers as follows [27]:

Let $S$ is the categorization of $R$ such that $S \subseteq R$. An approximation space $\boldsymbol{A}=(\square, S)$ will be denoted as: For a given $S=\left(x_{i}\right)_{i=1}^{n}$ the generalized projection $\Pi$ is defined: $\Pi(S)=\left\{\left(-\infty, x_{1}\right), x_{1},\left(x_{1}, x_{2}\right), x_{2}, \ldots\right\}$.

Notions $\bar{S}(x)$ remain as above. $Q(x)$ should be defined as $Q(x)=(-\infty, x\rangle$ what causes that definitions of its S-lower and S-upper approximations remain unchanged. Also definitions of real number $x$ remain the same as above. This extension of the Pawlak definitions leads us to the notion of rough real number.

\section{GREY SET}

There is a classification, which classifies sets into three different categories: White sets, Black sets and Grey sets,

Definition: For a set $A \subset U$, if its characteristic function value of each $x$ with respect to $A$ can be expressed with a single white number $v \in[0,1], \chi_{A}: \mathrm{U} \rightarrow[0,1]$, then A is a white set.

In fact, type-1 fuzzy sets can be considered as a special case of white sets. A crisp set is clearly a white set and it is not fuzzy at all, but a type-1 fuzzy set is still a white set although it is fuzzy compared with a crisp set.

Definition: For a set $A \subset U$, if it's characteristic function value of each $x$ with respect to $A$ can be expressed with a black number, then $A$ is a black set.

An element in a black set has a complete unknown characteristic function value, and it is opposite to a white set where we have complete knowledge about the characteristic function value of an element. Between the two extremes, a set with incomplete information about its characteristic function values is defined as a grey set.

Definition: For a set $A \subset U$, if the characteristic function value of $x$ with respect to $A$ can be expressed with a grey number $g_{A}^{ \pm}(x) \in \bigcup_{i=1}^{n}\left[a_{i}^{-}, a_{i}^{+}\right] \in D[0,1]^{ \pm}$

$\chi_{A}: \mathrm{U} \rightarrow D[0,1]^{ \pm}$Then $\mathrm{A}$ is a grey set.

Here, $D[0,1]^{ \pm}$refers to the set of all grey numbers within the interval $[0,1]$. The characteristic function here is a general expression; it does not exclude any relevant criteria in defining a set. Therefore, it can be replaced by probability function, membership function, possibility function and etc. For a white set, we know clearly the relationship between an element and a set. Obviously, a white set here is different from a crisp set in traditional sets. A white set has a clear relationship between the set and relevant elements, and that relationship is not necessarily a crisp relationship. If we replaced the characteristic function with a fuzzy membership function, then the white set would become a standard type-1 fuzzy set.

\subsection{Grey Number}

A grey number is a number with clear upper and lower boundaries but which has an unknown position within the boundaries. A grey number for the system is expressed mathematically as [28]:

$$
g^{ \pm} \in\left[g^{-}, g^{+}\right]=\left\{g^{-} \leq t \leq g^{+}\right\} \text {where } g^{ \pm} \text {is a grey }
$$

number, $\mathrm{t}$ is information, $g^{-}$and $g^{+}$are the lower and upper limits of the information.

Definition: (Degree of greyness of a grey number [29]). The significance of the interval to the unknown number represented by a grey number is called the degree of greyness. It can be expressed as $g^{\circ}\left(g^{ \pm}\right)=f\left(g^{-}, g^{+}\right)$

Here, $\mathrm{f}$ is a function to determine the significance of the interval to $g^{ \pm}$. Let $D=\left[d_{\text {min }}, d_{\text {max }}\right]$ be the domain of values represented by a grey number $g^{ \pm} \in\left[g^{-}, g^{+}\right]$then we have $d_{\text {min }} \leq g^{-}, g^{+} \leq d_{\max }$ and

$$
f\left(g^{-}, g^{+}\right)=\frac{g^{+}-g^{-}}{d_{\max }-d_{\min }} .
$$

There are two special situations for $g^{ \pm}$:

- If $g^{-}=g^{+}$, then we call $g^{ \pm}$a white number

- If $g^{-}=d_{\text {min }}, g^{+}=d_{\max }$ then we call $g^{ \pm}$a black number

Obviously, a white number is a single crisp value where we have full knowledge. On the contrary, a black number is a grey number we know nothing about it.

Definition: (Generalized grey numbers [30]). Let $g^{ \pm} \in \mathfrak{R}$ be an unknown real number within a union set of closed or open intervals $g^{ \pm} \in \bigcup_{i=1}^{n}\left[a_{i}^{-}, a_{i}^{+}\right]$

$$
\begin{gathered}
0<n<\infty, \quad a_{i}^{-}, a_{i}^{+} \in \mathfrak{R} \\
a_{i-1}^{+} \leq a_{i}^{-} \leq a_{i}^{+} \leq a_{i+1}^{+}
\end{gathered}
$$

Also $p_{i}$ is the probability for $g^{ \pm} \in\left[g^{-}, g^{+}\right]$if the following conditions hold
(1) $p_{i}>0$
(2) $\sum_{i=1}^{n} p_{i}=1$ 


\section{VAGUE SET}

Gau and Buehrer (1993) presented the theory of vague sets [8]. Chen (1995) presented the arithmetic operations between vague sets. Chen (1997) presented similarity measures between vague sets. A vague set $\tilde{A}$ in the universe of discourse $U$ is characterized by a truth-membership function $t_{\tilde{A}}$ and a false-membership function $f_{\tilde{\mathbf{A}}}$, where

$t_{\tilde{\mathbf{A}}}: U \rightarrow[0,1], \quad f_{\tilde{\mathbf{A}}}: U \rightarrow[0,1], \quad$ for $u_{\mathbf{i}} \in U, t_{\tilde{A}}\left(u_{\mathbf{i}}\right)$ is a lower bound of the grade of membership of $u_{\mathbf{i}}$ derived from the evidence for $u_{\mathbf{i}}$, $f_{\tilde{A}}\left(u_{\mathbf{i}}\right)$ is a lower bound of the negation of $u_{\mathbf{i}}$ derived from the evidence against $u_{\mathbf{i}}, t_{\tilde{A}}\left(u_{\mathbf{i}}\right)+f_{\tilde{A}}\left(u_{\mathbf{i}}\right) \leq 1$. The grade of membership of $u_{\mathbf{i}}$ in the vague set $\tilde{A}$ is bounded by a subinterval $\left[t_{\tilde{A}}\left(u_{\mathbf{i}}\right), 1-f_{\tilde{A}}\left(u_{\mathbf{i}}\right)\right]$ of $[0,1]$. The vague value $\left[t_{\tilde{A}}\left(u_{\mathbf{i}}\right), 1-f_{\tilde{A}}\left(u_{\mathbf{i}}\right)\right]$ indicates that the exact grade of membership $\mu_{\tilde{A}}\left(u_{\mathbf{i}}\right)$ is bounded by $t_{\tilde{A}}\left(u_{\mathbf{i}}\right) \leq \mu_{\tilde{A}}\left(u_{\mathbf{i}}\right) \leq 1-f_{\tilde{A}}\left(u_{\mathbf{i}}\right)$, where

$t_{\tilde{A}}\left(u_{\mathbf{i}}\right)+f_{\tilde{A}}\left(u_{\mathbf{i}}\right) \leq 1$. An example of a vague set $\tilde{A}$ in the universe of discourse $U$ is shown in Figure 9 [31].

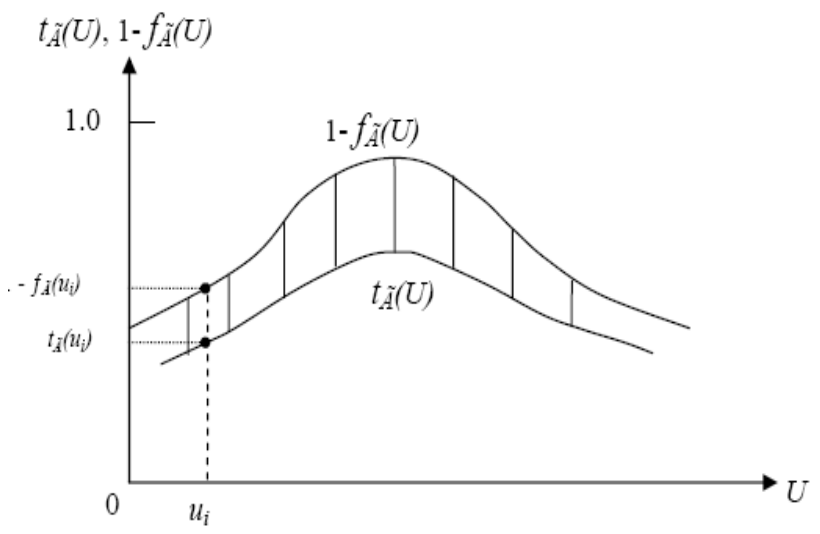

Fig 9: Vague Set

If the universe of discourse $U$ is a finite set, then a vague set $\tilde{A}$ of the universe of discourse $U$ can be represented as follows

[31]: $\tilde{A}=\sum_{i=1}^{n}\left[t_{\tilde{A}}\left(u_{\mathbf{i}}\right), 1-f_{\tilde{A}}\left(u_{\mathbf{i}}\right)\right] / u_{\mathbf{i}}$.

If the universe of discourse $U$ is an infinite set, then a vague set $\tilde{A}$ of the universe of discourse can be represented as [31]

$\tilde{A}=\int_{U}\left[t_{\tilde{A}}\left(u_{\mathbf{i}}\right), 1-f_{\tilde{A}}\left(u_{\mathbf{i}}\right)\right] / u_{\mathbf{i}} \quad u_{\mathbf{i}} \in U$. where the symbol $\int$ denotes the union operator.

\subsection{Vague Number}

A vague number is a vague subset in the universe of discourse $U$ that is both convex and normal. A vague set $\tilde{A}$ of the universe of discourse $U$ is called a normal vague set if $\exists u_{\mathbf{i}} \in U, \quad$ such that $1-f_{\tilde{A}}\left(u_{\mathbf{i}}\right)=1 . \quad$ That is $f_{\tilde{A}}\left(u_{\mathbf{i}}\right)=0$.

\subsection{Types of Vague Number}

There are many forms of vague numbers, among which the triangular vague number, trapezoidal vague number and Gaussian vague model are the most common ones

\subsubsection{Triangular vague number}

Definition [32]: a vague number $\tilde{A}$ is called Triangular vague number if

$$
\begin{gathered}
t_{\tilde{A}}(x)=\left\{\begin{array}{lc}
0 & x<a_{1} \\
\frac{x-a_{1}}{w\left(a_{2}-a_{1}\right)} & a_{1} \leq x<a_{2} \\
\frac{a_{3}-x}{w\left(a_{3}-a_{2}\right)} & a_{2} \leq x<a_{3} \\
0 & x>a_{3}
\end{array}\right. \\
1-f_{\tilde{A}}\left(u_{\mathbf{i}}\right)=\left\{\begin{array}{lc}
0 & x<a_{1} \\
\frac{x-a_{1}}{a_{2}-a_{1}} & a_{1} \leq x<a_{2} \\
\frac{a_{3}-x}{a_{3}-a_{2}} & a_{2} \leq x<a_{3} \\
0 & x>a_{3}
\end{array}\right.
\end{gathered}
$$

Where $w \in[1, \infty)$ this vague number is denoted by $\tilde{A}=\left(a_{1}, a_{2}, a_{3}, w\right)$

\subsubsection{Trapezoidal Fuzzy Number}

Definition [32]: vague number $\tilde{A}$ is called a trapezoidal vague number if

$$
t_{\tilde{A}}(x)=\left\{\begin{array}{lc}
0 & x<a_{1} \\
\frac{x-a_{1}}{w\left(a_{2}-a_{1}\right)} & a_{1} \leq x \leq a_{2} \\
\frac{1}{w} & a_{2} \leq x \leq a_{3} \\
\frac{a_{4}-x}{w\left(a_{4}-a_{3}\right)} & a_{3} \leq x \leq a_{4} \\
0 & x>a_{4}
\end{array}\right.
$$$$
1-f_{\tilde{A}}\left(u_{\mathbf{i}}\right)=\left\{\begin{array}{lc}
0 & x<a_{1} \\
\frac{x-a_{1}}{a_{2}-a_{1}} & a_{1} \leq x \leq a_{2} \\
1 & a_{2} \leq x \leq a_{3} \\
\frac{a_{4}-x}{a_{4}-a_{3}} & a_{3} \leq x \leq a_{4} \\
0 & x>a_{4}
\end{array}\right.
$$ 
Where $w \in[1, \infty)$ this vague number is denoted by $A=\left(a_{1}, a_{2}, a_{3}, a_{4}, w\right)$

\section{GRANULAR COMPUTING}

Fuzzy sets, interval analysis, rough sets, grey set, lntuitionistic fuzzy sets, and vague set are all constructs falling under the same umbrella of Granular Computing which has recently emerged as a coherent conceptual and algorithmic platform aimed at the representation and processing of information granules [33].

Granular computing $(\mathrm{GrC})$ is an emerging computing paradigm of information processing. It concerns the processing of complex information entities called information granules, which arise in the process of data abstraction and derivation of knowledge from information [34]. Generally speaking, information granules are collections of entities that usually originate at the numeric level and are arranged together due to their similarity, functionality, physical adjacency, indistinguishability, coherency, or the like [35].in the next subsection we introduce the proposed unified uncertain granular number.

\subsection{Unified Granular Number}

There are many forms of inexact granular numbers such as [36]: interval numbers, fuzzy numbers, rough numbers, vague numbers, grey numbers ....etc, most of current algorithms depend on certain data and exact numbers. There is an urgent need for new generations of algorithms which can handle uncertain data and inexact numbers of each type, which is challenge work. Some researchers resorted to create formulas of transformations among different representations of uncertain granular numbers; these transformations are still not adequate for all forms of numbers.

Even if we imagine that, we have all types of formulas of transformations between different representations of uncertain numbers, it's hard to solve types of problems which contain different types of uncertain granular numbers.

In this work we suggest a Unified Granular Number (U.G.N), that we call it G- Number to be as general formula for any uncertain granular number.

G- Number represents a higher level of abstract that hold common properties of all uncertain granular numbers, some details are not necessary to be considered but, the benefit from using G- number form is representing all types of uncertain number by same form.

Definition: G- Number is an extension of a regular number in the sense that it does not refer to one single value but rather it refers to a connected set of possible values. G- Number is a quantity whose value is imprecise, rather than exact as the case with "ordinary" (single-valued) numbers.

G-number X can be expressed by two factors which are: the center value of number $x$, and radius of number $\mathrm{r}_{\mathrm{x}}$ as in form $X=G\left(x, r_{x}\right)$

This formation of G-number guarantees representing the most common properties of uncertain granular number which are lower and upper bounded, and boundaries are known.

One may thinks that, the weakness point of using the past formula of unified granular number to represent various types of uncertain granular numbers is neglecting the membership function which is the main idea in some sets such as fuzzy set, the answer is: the philosophy of defuzzification is mainly depends on extracting a crisp value corresponding to the shape of fuzzy set and that what happened in using G-number. Even G- number formula represents the fuzzy number by using two parameters, one of them represents the center of domain (granular) and the other represents the radius of that domain (granular).

A modification is added to past formula of G- number by considering a new factor that is the area of granule which subtended between number graph and $\mathrm{x}$-axis to represent the weight of the number. Since $A(X)$ is the area of number $\mathrm{X}$ or the weight of number on its domain

$$
X=G \frac{\left(x, r_{x}\right)}{A(X)}
$$

Another form for unified granular number, this is granularratio-number or Gr-number. It could be formed by replacing the weight of the number by the ratio between that weight and length of its domain, or, by replacing the area which represents the weight of number by the ratio between the space area of number graph and the area of rectangle that contains the number location, that rectangle represents the full membership for the same domain in some cases like the case of fuzzy number.

\section{CONCLUSION}

There are many forms of inexact granular numbers such as: interval numbers, fuzzy numbers, rough numbers, vague numbers, grey numbers, and all these number falling under the same umbrella of Granular Computing. Unified granular number or G-number is a new representation for different types of inexact numbers; the main benefit from using Gnumber that, it gives us the ability to homogenate the heterogeneous types of uncertain granular numbers via converting each of them into the new representation of Gnumber, then the calculation on these numbers can be performed. This work opens the gate of using G-number to represent all types of uncertain granular numbers like rough numbers, vague numbers, grey numbers, and intervals.

\section{REFERENCES}

[1] Young, R.C., 1931" The algebra of many-valued quantities", Annals of Mathematics 31, pp. 260-290.

[2] Moore, R.E., 1979" Method and Application of Interval Analysis", Prentice Hall, London.

[3] Zadeh, L.A., 1965" Fuzzy sets", Information and Control 8, pp. 338-353.

[4] Zadeh, L.A., 1975" The concept of a linguistic variable and its application to approximate reasoning", Information Sciences, 8 pp 199-249.

[5] Pawlak, Z., 1982" Rough sets", International Journal of Information \& Computer Sciences 11, pp. 341-356.

[6] Deng, J.L., 1982" The Control problem of grey systems", System \& Control Letters 1(5), pp. 288-294.

[7] Atanassov, K. T., 1983" Intuitionistic fuzzy sets", VII ITKR's Session, pp. 391-396.

[8] Buehrer, D. J. 1992 "Vague logic: A first-order extension of interval probability theory" in Proc. IEEE Workshop Imprecise and Approximate Computation, Phoenix, AZ, pp. 83-87.

[9] Pedrycz, W., 2005 "Knowledge-based clustering: from data to information granules", J. Wiley, Hoboken, NJ.

[10] Thomas Jech, , 2003" Set Theory" Springer Monographs in Mathematics, Berlin, New York: Springer-Verlag, pp. 642-646. 
[11] Tiles, Mary, 2004" The Philosophy of Set Theory: An Historical Introduction to Cantor's Paradise". Dover Publications.

[12] Tobias Dantzig, 1930 "Number, the language of science”; New York, The Macmillan company.

[13] Sunaga, T., 1958 "Theory of interval algebra and its application to numerical analysis", Tokyo, Japan, 2, pp. $29-46$.

[14] Petkovi, M. and Petkovi, L., 1998 "Complex interval arithmetic and its applications", Wiley.

[15] Zadeh, L., 1996 "Fuzzy Sets, Fuzzy Logic, Fuzzy Systems", World Scientific Press, Singapore.

[16] Gerla, G., 2006"Effectiveness and Multivalued Logics". Journal of Symbolic Logic 71 (1): pp. 137-162.

[17] Zimmermann, H., 2001" Fuzzy set theory and its applications". Boston: Kluwer Academic Publishers.

[18] Novák, V.; Perfilieva, I. and Močkoř, J., 1999 "Mathematical principles of fuzzy logic", Dordrecht: Kluwer Academic.

[19] Buckley, J.J. 1991 "On the Hamacher sum of triangular fuzzy numbers", Fuzzy Sets and Systems 42 pp. 205212.

[20] Wang, C. and Chen, S., 2006 "A new method for appraising the performance of high school teachers based on simplified fuzzy number arithmetic operations". In: Proceedings of the 19th international conference on industrial, engineering\& other applications of applied intelligent systems. Annecy, France, pp 432-441.

[21] Chen, L., Cheng, C., 2005 "Selecting IS personnel using ranking fuzzy number by metric distance method". Eur J Oper Res 160(3), pp.803-820.

[22] Jerry M. Mendel, Robert I. and Bob John, 2002 "Type-2 Fuzzy Sets Made Simple”, IEEE, April 2002.

[23] Juan R. Castro, Oscar and Castillo, 2007 "Interval Type2 Fuzzy Logic for Intelligent Control Applications", IEEE.

[24] Pawlak, Z. 1992 "Rough sets: a new approach to vagueness," in: Fuzzy Logic for the Management of Uncertainty, John Wiley \& Sons, New York, pp. 105118.
[25] Pawlak, Z. 1994 "Hard and soft sets," in: Rough Sets, Fuzzy Sets and Knowledge Discovery, Springer-Verlag, London, pp. 130-135.

[26] Pawlak, Z., 1996" Rough sets, rough relations and rough functions", Fundamental Informatics, 27(2-3), 103-108.

[27] Marcin M., 2011 "Rough Numbers and Rough Regression", Lecture Notes in Computer Science Springer Moscow, Russia.

[28] Deng, J.L., 2002"The Foundation of Grey Systems Theory". Wuhan: The Press of the Central China University of Science and Technology.

[29] Lin, Y., Chen, M.Y. and Liu, S., 2004” Theory of grey systems: capturing uncertainties of grey information", The International Journal of Systems and Cybernetics 33(2), pp.196-218.

[30] Yang, Y. and Hinde,C. 2010 "A new extension of fuzzy sets using rough sets: R-fuzzy sets", Information Sciences 180 (3) pp. 354-365

[31] Watanabe, A., 2010 "Vague Quantity, Numerals, and Natural Numbers", the Dynamics of Complex Systems, 120(7), pp. 37-77.

[32] G. Beliakova, H. Bustinceb, D. Goswamic, U. Mukherjeed, N. Pale, 2011 "On averaging operators for atanassov's intuitionistic fuzzy sets", Information Sciences 181 (6) pp. 1116-1124.

[33] Pedrycz, W. and Gomide, F., 2007" Fuzzy systems engineering: toward human-centric computing", John Wiley, Hoboken, NJ.

[34] Pedrycz, W. and Valente de Oliveira, J., 2008 "A development of fuzzy encoding and decoding through fuzzy clustering", IEEE Transactions on Instrumentation and Measurement, 57, N.4, pp.829-837.

[35] Pedrycz, W. and Liu, X., 2009” Axiomatic fuzzy set theory and its applications", Springer Verlag, Berlin 244, pp. 2475-2486.

[36] Pedrycz, W., Loia, V. and Senatore, S. 2010 " Fuzzy clustering with viewpoints", IEEE Trans. on Fuzzy Systems, 161(1) pp. 56-74. 